\title{
Probing the dynamics of biological matter by elastic, quasi-elastic, and inelastic neutron scattering
}

\author{
Giorgio Schirò ${ }^{1, *}$ \\ ${ }^{1}$ Université Grenoble Alpes, CEA, CNRS, Institut de Biologie Structurale, F-38000, Grenoble, France
}

\begin{abstract}
The so-called function-structure-dynamics paradigm established that a close relationship links the way biological molecules work (function), their 3-dimensional organization (structure) and the changes of this organization in time (dynamics), which characterize biomolecules as highly dynamic objects. A typical example of protein dynamics is provided by protein reactions with substrates: equilibrium thermal fluctuations of protein structure are necessary to allow the access of substrates to the active site, where the functional reaction occurs. Neutron scattering is a powerful technique to study equilibrium protein structural dynamics. The incoherent structure factor, which is dominant in neutron scattering from biological matter, is related to the time-position self correlation function of protein/solvent nuclei. Here the basic theory of neutron scattering and the principles of the technologies used to measure it are described. Some selected applications of neutron scattering for investigating the structural dynamics of biological molecules are also reviewed.
\end{abstract}

\section{Theoretical background}

This section contains the basic formalism for describing neutron scattering in a quantitative way and forms the basis for understanding the following sections dedicated to neutron scattering from biological matter. We will show how the macroscopic physical quantities measured by neutron scattering are related to the microscopic state of the matter interacting with neutrons during an experiment. More detailed descriptions of the theory of neutron scattering can be found in the textbooks that have been used as the basis for the short description reported here [1-3].

The neutron is an electrically neutral nuclear particle with a mass $m_{n}=1.675 \times 10^{-27} \mathrm{~kg}$ and a spin $s=1 / 2$. The neutron does not live naturally in free form, but decays into a proton, an electron, and an antineutrino, with a lifetime $\tau \sim 886 \mathrm{~s}$, much longer than the time of a scattering event during an experiment, typically a fraction of a second. The neutron interacts with nuclei via the strong nuclear force and with magnetic moments via dipole-dipole coupling.

\footnotetext{
"Corresponding author: giorgio.schiro@ibs.fr
} 


\subsection{Scattering cross sections}

We first introduce the terms used to describe the scattering of a neutron beam. We define the flux of a neutron beam as

$$
\Psi=\frac{\text { number of neutrons incident on a surface per second }}{\text { surface area perpendicular to the neutron beam direction }}
$$

The central parameter to describe the interaction of a neutron beam with matter is the neutron scattering cross section $\sigma$ defined as

$$
\sigma=\frac{1}{\Psi} \cdot \text { number of neutrons scattered per second }
$$

which has the units of an area. The angular dependence of the scattered neutrons is the most important aspect of the scattering process. To describe this we use the so-called differential scattering cross section, defined as:

$$
\frac{d \sigma}{d \Omega}=\frac{1}{\Psi} \cdot \frac{\text { number of neutrons scattered per second in the solid angle } d \Omega}{d \Omega}
$$

We consider now the possibility that, during the scattering process, incident neutrons exchange part of their energy with the target nuclei, which is defined as inelastic scattering. Conversely, if incident neutrons are scattered with no energy exchange we refer to as elastic scattering. In order to describe the inelastic scattering process we have to take into account the energy dependence of the scattered neutrons. We define the double differential scattering cross section as:

$$
\frac{d^{2} \sigma}{d \Omega d E}=\frac{1}{\Psi} \cdot \frac{n . \text { of neutrons scattered per second in solid angle } d \Omega \text { with energies }[E ; E+d E]}{d \Omega}
$$

In the neutron scattering experiments that will be described here, only the strong nuclear interaction between neutrons and nuclei in the sample is considered. We define the state of the incoming wave as:

$$
\mid \psi_{i}>\propto \exp \left(i \widehat{k_{0}} \cdot \widehat{r}\right)
$$

where $\widehat{k_{0}}$ is the wave vector of the incoming neutron, $\widehat{r}$ is its position vector and the symbol $\propto$ indicates that the normalization factor has been omitted. We express the final neutron state at the detector as (a superposition of) plane waves, each with the form:

$$
\mid \psi>\propto \exp (i \widehat{k} \cdot \widehat{r})
$$


We describe the interaction responsible for the scattering by the operator $\widehat{V}$. By using the Fermi Golden Rule, which defines the rate of change between the neutron in the incoming state and that in the final state (we omit here some straightforward derivations, in particular the calculation of the density of states), we can obtain an expression for the differential scattering cross section:

$$
\frac{d \sigma}{d \Omega} \propto \frac{\widehat{k}}{\hat{k}_{0}}\left(\frac{m_{n}}{2 \pi \hbar^{2}}\right)^{2} \quad\left|<\psi_{0}\right| \widehat{V}|\psi>|^{2}
$$

To calculate the cross section we need an explicit form for the interaction operator $\widehat{V}$. The interaction between the neutron and the nuclei can be approximated by the Fermi pseudopotential defined as:

$\widehat{V}=\frac{2 \pi \hbar^{2}}{m_{n}} b_{N} \delta\left(\widehat{r}-\widehat{r_{N}}\right)$

where $\widehat{r_{N}}$ is the position vector of the target nucleus in the laboratory frame. Here the main parameter is the scattering length $b_{N}$. The spatial $\delta$ function accounts for the short range of the strong nuclear forces and gives a sufficient description of scattering of thermal neutrons. For a system composed by a single nucleus, we can now calculate the scattering cross section by using Eq. (7). We start by calculating the matrix element:

$$
\begin{gathered}
\mid\left\langle\psi_{0}|\widehat{V}| \psi>\right|=\frac{2 \pi \hbar^{2}}{m_{n}} b_{N} \int \exp \left(i \widehat{k_{0}} \cdot \widehat{r}\right) \delta\left(\widehat{r}-\widehat{r_{N}}\right) \exp (-i \widehat{k} \cdot \widehat{r}) d^{3} r= \\
=\frac{2 \pi \hbar^{2}}{m_{n}} b_{N} \exp \left(\hat{i} \cdot \widehat{r_{N}}\right)
\end{gathered}
$$

where $\widehat{Q}=\widehat{k_{0}}-\widehat{k}$ is the momentum transfer. Inserting Eq. (9) into Eq. (7), we obtain:

$$
\frac{d \sigma}{d \Omega}=b_{N}^{2}
$$

As already mentioned, during the scattering event generally neutrons can transfer energy to or absorb energy from the scattering system. We indicate the neutron energy transfer by:

$$
\hbar \omega=E_{i}-E \frac{\hbar^{2}\left(k_{0}^{2}-k^{2}\right)}{2 m_{n}}
$$

In this case, Eq. (7) assumes the form: 


$$
\frac{d \sigma}{d \Omega} \propto \frac{\widehat{k}}{\widehat{k}_{0}}\left(\frac{m_{n}}{2 \pi \hbar^{2}}\right)^{2}\left|<\psi_{0}, E_{0}\right| \widehat{V}|\psi, E>|^{2} \delta\left(E-E_{0}-\hbar \omega\right)
$$

where the $\delta$ function expresses the energy conservation in the scattering event. In a system of $\mathrm{N}$ nuclei, possibly with different scattering lengths, interferences between scattered waves from different nuclei have to be considered. We will neglect here absorption and multiple scattering effects, that can actually be minimized in certain experimental conditions, but also quantitatively taken into account in case such conditions cannot be realized [4]. The differential cross section reads as:

$$
\frac{d^{2} \sigma}{d \Omega d \omega} \propto \frac{\widehat{k}}{\widehat{k}_{0}} \sum_{i, j} b_{i} b_{j} \frac{1}{2 \pi} \int_{-\infty}^{+\infty} e^{i \omega t}\left\langle\exp \left(-i \widehat{Q} \cdot \widehat{r}_{i}(0)\right) \exp \left(i \widehat{Q} \cdot \widehat{r}_{j}(t)\right)\right\rangle d t
$$

where the indexes $i$ and $j$ are over all nuclei, $\widehat{r}_{i}(t)$ and $\widehat{r}_{j}(t)$ denote the position of nuclei $i$ and $j$ at time $t$, and \langle\rangle indicates the ensemble average. We have also assumed here that the system is stationary.
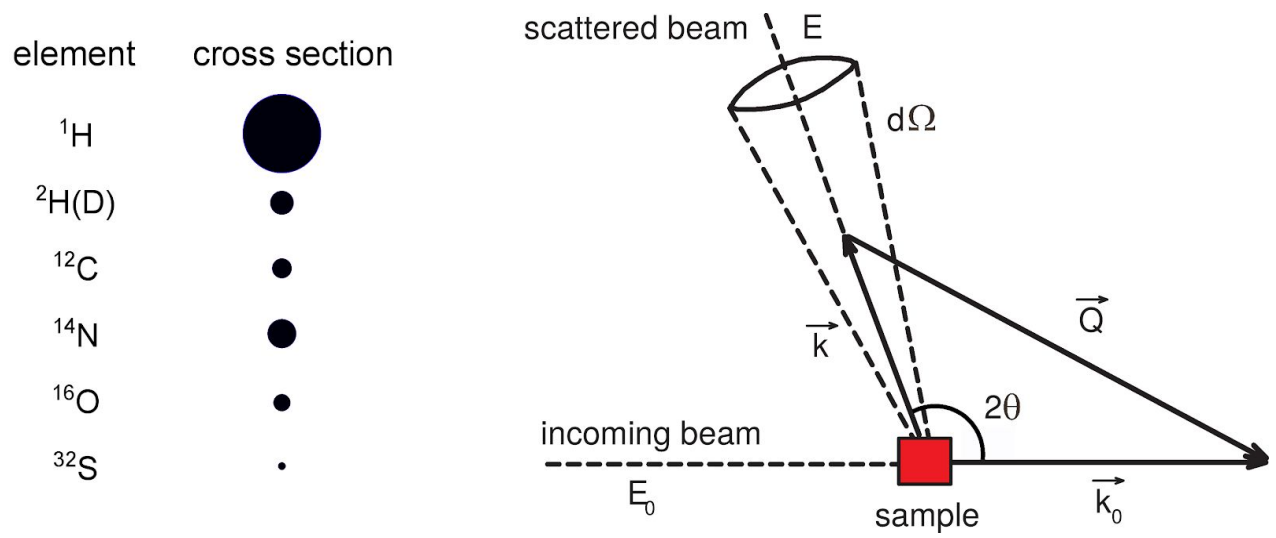

Fig. 1. Left: neutron scattering total cross sections of the most abundant elements present in biological molecules (the area of black circles is proportional to the total cross sections). Right: scheme of the scattering process. An incoming neutron with a wave vector $\widehat{k_{0}}$ interacts with nuclei in the sample and is scattered with a wave vector $\widehat{k}$. The fundamental parameters of the process are the momentum transfer $\widehat{Q}=\widehat{k_{0}}-\widehat{k}$ and the exchanged energy $E-E_{0}$.

In general, the neutron scattering length $b$ varies, the variation being due to the heterogeneity originating from isotopes of the same element, or from different elements. This represents a static disorder that, in the case of a macroscopic sample, can be treated in the same way as a time-dependent disorder (which corresponds to the ergodic hypothesis), since we can assume that the sample is large enough to essentially represent an ensemble average. We assume that the scattering length at site $i$ has the stochastic value $b=\bar{b} \pm \delta b$, where $\bar{b}$ is shorthand for the average of $b$ and the deviation from the average is assumed independent from specific site and 
is defined as $\delta b=\sqrt{\overline{b^{2}}-\bar{b}^{2}}$. Generally, the explicit average notation $\bar{b}$ drops, and the symbol $b$ is used to indicate the average scattering length of a given isotope or element. The total cross section defined in Eq. (13) can then be decomposed into two different terms; one is called coherent and the other one incoherent and are defined as:

$$
\begin{aligned}
\frac{d^{2} \sigma}{d \Omega d \omega} & =\left(\frac{d^{2} \sigma}{d \Omega d \omega}\right)_{i n c}+\left(\frac{d^{2} \sigma}{d \Omega d \omega}\right)_{c o h} \propto \\
& \left.\propto \frac{\widehat{k}}{\widehat{k}_{0}} \overline{\left(b^{2}\right.}-\bar{b}^{2}\right) \sum_{i} \frac{1}{2 \pi} \int_{-\infty}^{+\infty} e^{i \omega t}\left\langle\exp \left(-i \widehat{Q} \cdot \widehat{r}_{i}(0)\right) \exp \left(i \widehat{Q} \cdot \widehat{r}_{i}(t)\right)\right\rangle d t+ \\
& +\frac{\widehat{k}}{\widehat{k}_{0}} \bar{b}^{2} \sum_{i, j} \frac{1}{2 \pi} \int_{-\infty}^{+\infty} e^{i \omega t}\left\langle\exp \left(-i \widehat{Q} \cdot \widehat{r}_{i}(0)\right) \exp \left(i \widehat{Q} \cdot \widehat{r}_{j}(t)\right)\right\rangle d t= \\
& =\frac{\widehat{k}}{\widehat{k}_{0}}\left(\bar{b}^{2}-\bar{b}^{2}\right) S_{i n c}(\widehat{Q}, \omega)+\frac{\widehat{k}}{\widehat{k}_{0}} \bar{b}^{2} S_{c o h}(\widehat{Q}, \omega)
\end{aligned}
$$

The terms $S_{\text {inc }}(Q, \omega)$ and $S_{c o h}(Q, \omega)$ are known as incoherent and coherent dynamic structure factors, respectively. $S_{c o h}(Q, \omega)$, which results from the coherent superposition of the scattering from pairs of scatterers, accounts for the correlation among positions of different nuclei, whereas $S_{i n c}(Q, \omega)$, which does not contain any phase information, accounts for the correlation among positions of a single nucleus at different times. The difference between coherent and incoherent scattering can be easily explained if we consider the elastic scattering from a crystal, i.e. from atoms not moving. The elastic coherent scattering as a function of the scattering angle leads to Bragg peaks containing information about the relative position of the scattering nuclei. Conversely, the elastic signal originating from incoherent scattering is flat and the only information we can extract from it is the number of scatterers. We consider now a system composed by moving atoms. In this case the wavefronts emitted at different times from the same particle are coherent and lead to interference among the different scattered waves. The incoherent signal then provides information on the single particle motions. Incoherent and coherent cross sections are usually defined as:

$$
\begin{aligned}
& \sigma_{i n c}=4 \pi\left(\bar{b}^{2}-\bar{b}^{2}\right) \\
& \sigma_{c o h}=4 \pi \bar{b}^{2} .
\end{aligned}
$$

The scattering cross sections vary in an irregular manner as a function of element type and isotope mass number. The case of the hydrogen atom is particularly interesting for the study of soft matter and, in particular, of biological matter. Its nucleus is composed of a single proton, with spin $1 / 2$. The incoherent scattering cross section of the hydrogen atom is much larger than the incoherent or coherent scattering cross sections of all other elements. This characteristic makes incoherent neutron scattering an ideal tool for the study of individual motions in materials abundant in hydrogen. In the case of deuterium $\left({ }^{2} \mathrm{H}\right.$ or $\left.\mathrm{D}\right)$, the relative part of incoherent scattering over the total scattering is strongly reduced with respect to hydrogen ${ }^{1} \mathrm{H}$. 
In view of both the natural abundance of hydrogen and its large incoherent cross section, if compared with other elements in biological molecules (see Fig. 1), it is evident why neutron scattering represents a technique ideal to study the dynamics of biological systems. In particular, as hydrogens are abundant and uniformly distributed in proteins, this technique gives average information on the dynamics of a protein, as we will see in the next sections.

\subsection{Scattering functions}

We now want to relate the observables describing the neutron scattering event, as introduced in the previous section, with the microscopic properties of the matter that interacts with the neutron beam. A valid approach is to use so-called correlation functions. The theory of neutron scattering from a physical system in terms of the space-time correlation function $G(\widehat{r}, t)$ has been developed by L. van Hove [5]. This formalism is very useful for interpreting scattering data for interacting systems (such as liquids) where the exact formulation of the scattering is extremely complicated. The pair-correlation function describes the position of nuclei in space and time, and for $\mathrm{N}$ nuclei it is given by:

$$
G(\widehat{r}, t)=\frac{1}{N} \sum_{i, j}^{N}\left\langle\delta\left(\widehat{r}+\widehat{r}_{i}(0)-\widehat{r}_{j}(t)\right)\right\rangle
$$

If $j=i$, the pair correlation function is called self pair correlation function. The space Fourier transform of the pair correlation function $G(\widehat{r}, t)$ is called intermediate scattering function:

$$
\begin{aligned}
I(\widehat{Q}, t) & \propto \int_{-\infty}^{+\infty} G(\widehat{r}, t) \exp (i \widehat{Q} \cdot \widehat{r}) d r= \\
& =\frac{1}{N} \sum_{i, j}^{N}\left\langle\exp \left(-i \widehat{Q} \cdot \widehat{r}_{i}(0)\right) \exp \left(i \widehat{Q} \cdot \widehat{r}_{j}(t)\right)\right\rangle
\end{aligned}
$$

The time Fourier transform of the intermediate scattering function leads to the so-called dynamic structure factor:

$$
\begin{aligned}
S(\widehat{Q}, \omega) & \propto \int_{-\infty}^{+\infty} I(\widehat{Q}, t) \exp (-i \omega t) d t= \\
& =\int_{-\infty}^{+\infty} \int_{-\infty}^{+\infty} G(\widehat{r}, t) \exp (i \widehat{Q} \cdot \widehat{r}) d r \exp (-i \omega t) d t .
\end{aligned}
$$

Each of the functions $G(\widehat{r}, t), S(\widehat{Q}, \omega), I(\widehat{Q}, t)$ can equally describe the properties of the observed sample; however, only $S(\widehat{Q}, \omega)$ and $I(\widehat{Q}, t)$ are the quantities that can be directly accessed by neutron scattering. Eqs. (17) and (18) then relate the quantities measured by neutron scattering $(S(\widehat{Q}, \omega)$ and $I(\widehat{Q}, t))$ with the microscopic description of the sample in terms of the probability for nuclei in the sample to be found in a given position at a given time $(G(\widehat{r}, t))$. It is evident from Eq. (17) that $I(\widehat{Q}, t)$ can be divided into a coherent and an incoherent part, as we did above (Eq. (14)) for the dynamic structure factor. As discussed at the end of the previous section, the neutron scattering from biological matter is often dominated by the incoherent signal. Indeed, about $50 \%$ of the atoms in biological matter are composed of hydrogen atoms, which usually corresponds to more than $70 \%$ of incoherent signal over the 
total scattering signal in amorphous (i.e. non crystalline) samples. In the next sections we will then focus on the incoherent components of $S(\widehat{Q}, \omega)$ and $I(\widehat{Q}, t)$.

\subsection{Separation of different motions}

The motion of a scattering nucleus can be described as the superposition of translational, rotational and vibrational motions. The position of the nucleus can be then separated into three terms, for translational $\left(\widehat{r}_{t r}(t)\right)$, rotational $\left(\widehat{r}_{r o t}(t)\right)$ and vibrational $\left(\widehat{r}_{v i b}(t)\right)$ motions which as a first approximation can be assumed to be dynamically decoupled:

$\widehat{r}(t)=\widehat{r}_{t r}(t)+\widehat{r}_{r o t}(t)+\widehat{r}_{v i b}(t)$

Subsequently the incoherent parts of the functions $I(\widehat{Q}, t)$ and $S(\widehat{Q}, \omega)$ can be written as:

$$
\begin{aligned}
& I_{i n c}(\widehat{Q}, t)=I_{i n c}^{t r}(\widehat{Q}, t) \cdot I_{i n c}^{r o t}(\widehat{Q}, t) \cdot I_{i n c}^{v i b}(\widehat{Q}, t) \\
& S_{i n c}(\widehat{Q}, \omega)=S_{i n c}^{t r}(\widehat{Q}, \omega) \otimes S_{i n c}^{r o t}(\widehat{Q}, \omega) \otimes S_{i n c}^{v i b}(\widehat{Q}, \omega)
\end{aligned}
$$

where the symbol $\otimes$ denotes the convolution operation.

\subsection{Elastic, quasi-elastic and inelastic scattering}

Dividing the incoherent intermediate scattering function into time-independent and time-dependent parts as follows:

$I_{i n c}(\widehat{Q}, t)=I_{i n c}(\widehat{Q}, \infty)+I_{i n c}^{i n e l}(\widehat{Q}, t)$

and taking its Fourier transform (see Eq. (18)) leads to

$$
S_{i n c}(\widehat{Q}, \omega)=I_{i n c}(\widehat{Q}, \infty) \cdot \delta(\omega)+S_{i n c}^{\text {inel }}(\widehat{Q}, \omega)
$$

where $I_{i n c}(\widehat{Q}, \infty) \cdot \delta(\omega)$ is a component purely elastic and $S_{\text {inc }}^{\text {inel }}(\widehat{Q}, \omega)$ accounts for the energy transfer between scattered neutrons and sample nuclei and includes the information about the microscopic dynamics of the sample that are reflected on the energy-dependence of the structure factor.

$S_{\text {inc }}^{\text {inel }}(\widehat{Q}, \omega)$ contains contributions at small energy exchanges that can be separated from the inelastic scattering above in the so-called quasi-elastic term. This term originates from the diffusive motions of the scattering nuclei and can be described by $S_{i n c}^{t r}(\widehat{Q}, \omega)$ and $S_{i n c}^{r o t}(\widehat{Q}, \omega)$. The vibrational motions described by $S_{i n c}^{v i b}(\widehat{Q}, \omega)$ often produce inelastic spectra outside of the quasi-elastic region and originate from the vibrational levels of molecules. Since the vibrations and librations of the particles are often much faster (i.e. they have higher energy exchange) than the diffusive motions, it is reasonable to introduce them as an inelastic background in the quasi-elastic region. Thus the quasi-elastic incoherent scattering function can be written as:

$$
S_{i n c}^{q e}(\widehat{Q}, \omega)=\exp \left(-\left\langle u^{2}\right\rangle Q^{2}\right)\left[I_{i n c}^{d i f}(\widehat{Q}, \infty) \cdot \delta(\omega)+S_{i n c}^{d i f}(\widehat{Q}, \omega)+S_{i n c}^{i}(\widehat{Q}, \omega)\right]
$$

where the exponential factor, known as the Debye-Waller factor, accounts for the decrease in intensity due to vibrations, $S_{i n c}^{i}(\widehat{Q}, \omega)$ is the inelastic background in the quasi-elastic region, 
$S_{\text {inc }}^{\text {dif }}(\widehat{Q}, \omega)$ represents the quasi-elastic term and $I_{i n c}^{\text {dif }}(\widehat{Q}, \infty)$ is called elastic incoherent structure factor (EISF) and is the fraction of the total quasi-elastic intensity contained in the purely elastic peak. In the experimental data (see Fig. 2) the elastic scattering appears, instead of as an infinitely sharp line, as a peak shaped curve with an energy width corresponding to the instrumental resolution. This width defines the magnitude of the timescale over which the diffusive motions are observable. If the diffusive motions are significantly slower than the time window defined by the width of the instrumental resolution, their quasi-elastic contribution cannot be distinguished from the elastic peak. On the other hand, if the diffusive motions are much faster than the timescale determined by the instrumental resolution, the quasi-elastic component will be a very broad and flat term. If the elastic component and the wider quasi-elastic component can be separated, the EISF is a measurable quantity, evaluated from the ratio:

$$
\operatorname{EISF}(\widehat{Q})=\frac{I^{e l}(\widehat{Q})}{I^{e l}(\widehat{Q})+I^{q e}(\widehat{Q})}
$$

where $I^{e l}(\widehat{Q})$ and $I^{q e}(\widehat{Q})$ are the integrated (in the $\omega$ space) intensities of the elastic and quasi-elastic components of the spectra, respectively. While the width of the quasi-elastic component can reveal information on the characteristic times of the diffusive motions, the $Q$-dependent EISF provides information about the nature of the diffusive motion itself.

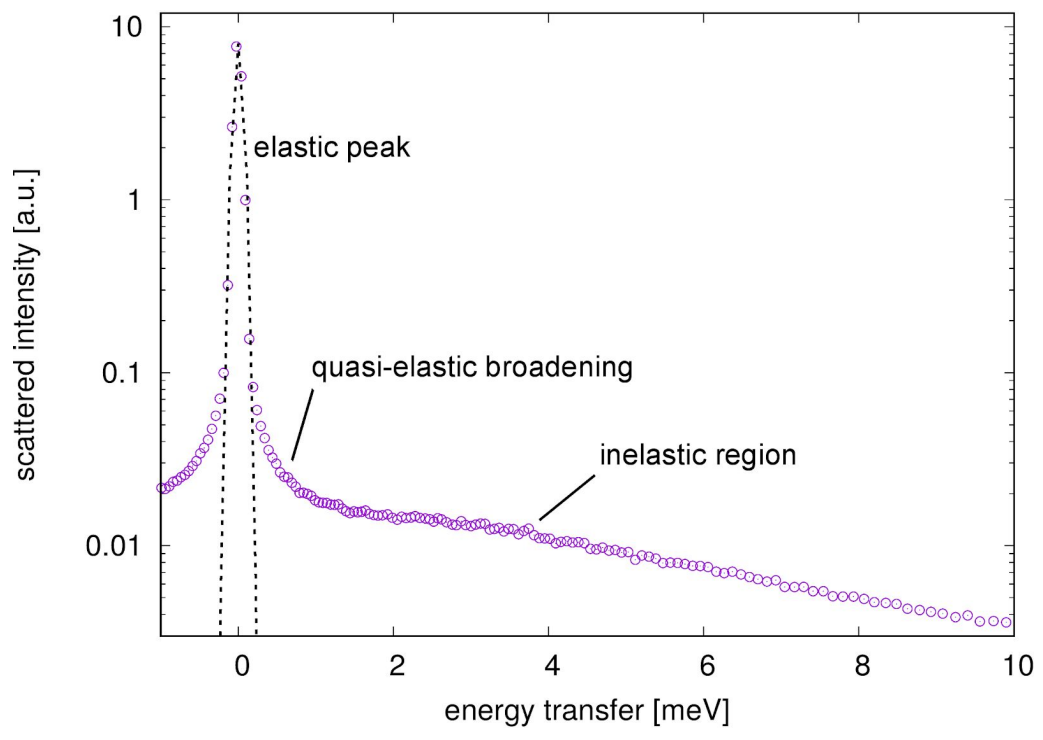

Fig. 2. Neutron scattering spectrum of a $\mathrm{D}_{2} \mathrm{O}$-hydrated myoglobin powder at the temperature $\mathrm{T}=260 \mathrm{~K}$ measured on the time-of-flight spectrometer IN6 (Institut Laue-Langevin, Grenoble, France). The dashed line indicates the lineshape of the instrumental resolution function.

\section{Spectrometers for neutron scattering}

As discussed in the previous section, a neutron scattering experiment consists in determining the status of a neutron beam after interaction with the sample in terms of neutron energy and momentum, provided that the same quantities are known for the neutron beam before the 
interaction. Depending on the way these quantities are measured, different neutron spectroscopy techniques can be distinguished. If the change in the direction of scattered neutrons is generally defined by the position of detectors relative to the incident beam direction, the change in neutron energy can be indeed measured using different approaches, known as backscattering, time-of-flight and spin-echo. A fourth approach, called three-axis, will not be discussed here. Every technique is characterized by different energy and momentum transfers that can be measured, which in turn define the time and space scales of the structural dynamics that can be probed, as represented in an approximate way in Figure 3. The exact ranges are of course defined by the details and the technical parameters of a given spectrometer.

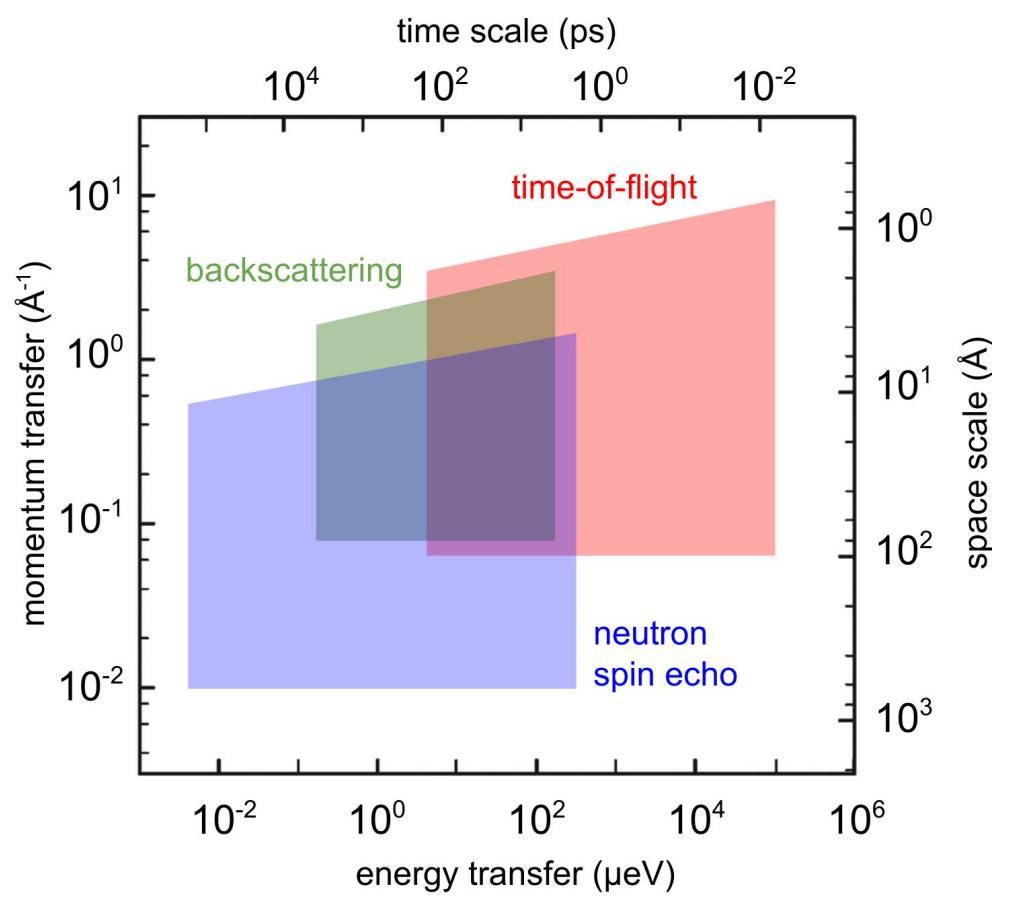

Fig. 3. The energy and momentum transfer ranges probed by the different neutron spectroscopy techniques provide access to molecular motions at different space and time scales.

\subsection{Neutron backscattering spectroscopy}

Neutron backscattering spectroscopy uses the principle of backscattering, which refers to the reflection of a neutron wave back to the same direction it comes from. This technique makes use of a Bragg angle of $90^{\circ}$ to analyze and select the wavelength of neutrons after interaction with the sample, which allows to obtain a high energy resolution, even below $1 \mu \mathrm{eV}$. Since the Bragg angle in the analyzer cannot be changed to select different energies, different energies transfers are achieved by varying the energy of the incoming beam. This is implemented either changing the lattice constant in the monochromator crystal by changing its temperature or by Doppler effect obtained by periodically translating monochromator crystal along the reflection direction. The disadvantage of these methods for changing the energy transfer is the inherent limited energy range that can be explored. Examples of neutron backscattering spectrometers 
are SPHERES at the Maier-Leibnitz Zentrum in Garching (Germany) and IN16B at the Institut Laue-Langevin in Grenoble (France).

\subsection{Time-of-flight spectroscopy}

In the time-of-flight approach the energy of the scattered neutrons is determined by measuring their kinetic energy after interaction with the sample. The kinetic energy is calculated using the measurement of the time neutrons take to travel along the distance between the sample and the detector. The same principle can be used for selecting the energy of the neutron beam impinging on the sample, by means of a system of synchronized rotating choppers. Examples of time-of-flight spectrometers are TOFTOF at the Maier-Leibnitz Zentrum in Garching (Germany) and IN5 at the Institut Laue-Langevin in Grenoble (France).

\subsection{Neutron spin-echo spectroscopy}

Neutron spin-echo spectroscopy is the only neutron technique that enables accessing the dynamics of nuclei in the sample directly in the time domain. The main difference with respect to the other methods described above is that neutron spin-echo provides a direct estimation of the energy exchange, instead of calculating it by subtracting the neutron energy after interaction with the sample to energy before the interaction. The main idea at the basis of neutron spin-echo is to use Larmor precession of polarized neutrons travelling inside a magnetic field as an internal clock. Neutrons travel in identical magnetic fields before and after interacting with the sample and the spin orientation is inverted at the sample position. In elastic conditions, neutron polarization is restored at the outcome of the second magnetic field, otherwise, if energy has been exchanged with the sample, a change in the polarization will be measured, from which the inelastic scattering can be calculated in terms of the intermediate scattering function $I(\widehat{Q}, t)$. Examples of neutron spin-echo spectrometers are the NGA spectrometer at the NIST Center for Neutron Research (US) and IN15 at the Institut Laue-Langevin in Grenoble (France).

\section{Neutron scattering probes biomolecular dynamics}

In this section some applications of neutron scattering to investigate the dynamical behavior of biological matter are reviewed. Depending on the physical state of the sample and on the space-time scale probed by a given neutron spectrometer, the complexity of the structural dynamics contributing to the neutron scattering signal makes the data interpretation more or less challenging. In the case of proteins, for example, assuming that the $\mathrm{H}_{2} \mathrm{O} \rightarrow \mathrm{D}_{2} \mathrm{O}$ exchange in the buffer minimizes the solvent contribution to the scattering signal, the remaining signal arises from different classes of molecular motions (see Fig. 4). Local fluctuations at the side-chains and backbone level characterize the dynamics in the ps-ns time scale; internal dynamics are also composed by relative motions of protein domains, which can occur from ps up to $\mu$ s; finally, roto-translational diffusive motions of the entire macromolecules can be detected in the ns up to ms time scale, depending on the space scale probed by the neutron spectrometer and on the molecular weight of the macromolecule. The use of hydrated protein powders, where all motions but the internal dynamics are essentially suppressed, is a way to simplify the data interpretation and focus only on internal dynamics. 
In this section three different examples have been selected that illustrate the use of elastic, quasi-elastic and inelastic neutron scattering for studying the behaviour of biomolecules in terms of dynamical processes at a molecular level:

1) the role of hydration water in triggering the onset of functional motions in soluble proteins

[6] and how this can be implicated in protein pathological states [7];

2) the collective dynamics of lipid membranes as a function of temperature [8];

3 ) the diffusive dynamics of proteins in crowded conditions mimicking the cellular context $[9,10]$.

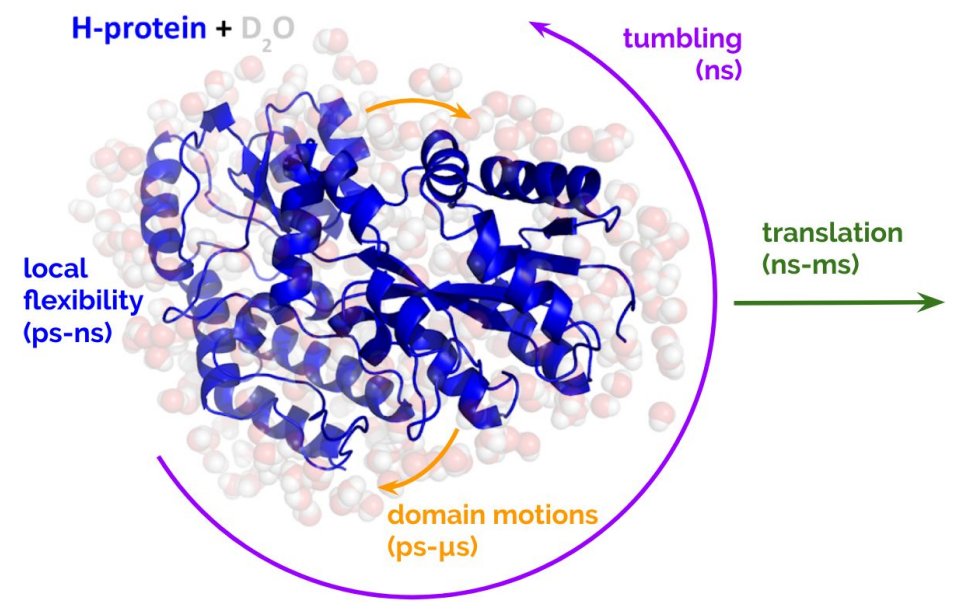

Fig. 4. Different classes of molecular motions characterize biological macromolecules: local side-chains and backbone fluctuations occurring in the ps-ns time scale, internal domain motions (ps- $\mu \mathrm{s}$ ) and roto-translational motions of the entire molecule, occurring in the ns up to ms time scale, depending on the probed space scale and on the size of the macromolecule.

\subsection{Quasi-elastic neutron scattering reveals the role of hydration water in protein dynamics}

Biological macromolecules such as soluble proteins are surrounded by a layer of water molecules (the so-called hydration water) interacting with the macromolecule surface. The active role of hydration water in macromolecular functions such as enzyme reactions, molecular recognition, allostery, and charge transfer is now largely recognized. In most completely dry proteins biological activity is lost, while it is recovered when the first hydration layer is present, thus making a protein together with its first hydration layer the biologically active entity. The interaction between protein and water molecules is essentially of electrostatic nature, with an extended hydrogen-bonded network connecting protein and water. The fluctuations of such a network are accompanied by breakage and formation of water-protein hydrogen bonds and allow for functionally important protein motions. The nature of the coupling between water and protein dynamics is a matter of extensive debate [11]. A way to tease apart the complex ensemble of water-protein motions is to extend experiments down to cryo-temperatures. When lowering the temperature below $\sim 240 \mathrm{~K}$ hydrated proteins undergo a transition where the large-amplitude anharmonic motions on the picosecond timescale, which are related to 
functional motions, are frozen. The origin of this transition, which has been named protein dynamical transition [12] and is paralleled by a transition in the picosecond motions of hydration water at a similar temperature [13], is controversially debated in the biophysical community [11]. However, there is a general agreement about the primary role played by hydration water dynamics in both protein dynamics and function. Indeed, when the hydration level is lowered below monolayer coverage both the dynamical transition and the biological activity of a protein are suppressed [14].

As discussed above, the incoherent scattering cross-section of a hydrogen atom is larger by about two orders of magnitude than that of all other atoms in a protein (see Fig. 1), including its isotope deuterium, and thus dominates the neutron scattering signal in non-crystalline protein samples. Replacing all hydrogen atoms in a protein by deuterium (a procedure called perdeuteration) is then a way to enhance the contribution from hydration water dynamics to the incoherent scattering signal and minimize that from protein dynamics. In perdeuterated proteins put in the form of freeze-dried powder hydrated at a level of $\sim 0.4$ gram of $\mathrm{H}_{2} \mathrm{O}$ per gram of protein, corresponding roughly to a monolayer coverage of the protein surface, more than $70 \%$ of the incoherent neutron scattering signal comes from hydration water.

In the work reviewed in this section [6] hydration water dynamics has been studied on the surface of the globular Maltose Binding Protein (MBP) and of the intrinsically disordered human protein tau. By combining protein perdeuteration and quasi-elastic neutron scattering, evidence has been provided that the onset of translational water motions on tau and MBP occurs at the same temperature where the proteins undergo the dynamical transition. The diffusive dynamics of a water molecule can be described, in the time scale probed by neutron scattering, as a translational motion of its centre-of-mass and a rotation around the centre-of-mass. The model used to analyze experimental data assumes that water molecules either translate, rotate or remain immobile. Indeed, the fitting quality has been proved to improve with a model function where the translational and rotational terms are added rather than convoluted ( $\chi^{2}$ at least $20 \%$ higher on average, but even $50 \%$ higher at temperatures above $250 \mathrm{~K}$ ). This can be explained by the fact that within the ns-ps time scale probed by the neutron spectrometer two populations of water molecules exist with different dynamical properties: one formed by water molecules strongly interacting with the protein surface and/or inside protein docking sites (represented by the rotational term) and another one formed by less hindered water molecules whose rotational motion is faster than the time window accessible by the neutron spectrometer (represented by the translational term). Such heterogeneity of water motions in the first hydration shell has been also observed by other techniques and on different time scales [15].

A novelty of the work reviewed here [6] was also the global fitting approach adopted, in which the distance between neutron experimental data and the model function (i.e. the $\chi^{2}$ function) was minimized in the two-dimensional space defined by momentum transfer and energy transfer. Compared to the fitting procedure usually adopted in the literature (i.e. fitting the experimental data in the energy transfer domain at every momentum transfer value and then analyzing the momentum transfer dependence of the fitting parameters), the approach proposed in [6] allows from one hand to minimize the number of free parameters and from the other hand to identify dynamical contributions to the quasi-elastic spectrum arising from confined motions (such as rotational motions). These contributions have indeed a typical length scale (like, in the present case, the radius of a rotational motion defined by the $\mathrm{H}-\mathrm{O}$ distance in the water molecule) so that their intensity depends on the momentum transfer and can vary significantly in its accessible range. From the global fit analysis, for each temperature point fractions of the 
total scattering intensity originating from water molecules that undergo translational or rotational diffusion, or that remain immobile have been then extracted. The plots in Fig. 5 show these fractions as a function of temperature for both tau and MBP. The translational fraction displays a steep increase above $\sim 240 \mathrm{~K}$, while the rotational one starts increasing at $\sim 200 \mathrm{~K}$ and reaches a plateau at $\sim 250 \mathrm{~K}$ (see Fig. 5). Up to $\sim 200 \mathrm{~K}$, nearly all water molecules are detected as immobile on the timescale determined by the energy resolution of the neutron spectrometer. With the resolution used in this work a molecule is defined as "immobile" when its motion is slower than $\sim 2$ ns. A full population of such "immobile" water molecules up to $\sim 200 \mathrm{~K}$ is in agreement with the presence of a calorimetric glass transition of hydration water at $\sim 170 \mathrm{~K}$, as actually measured in hydrated powders of other globular proteins [16].

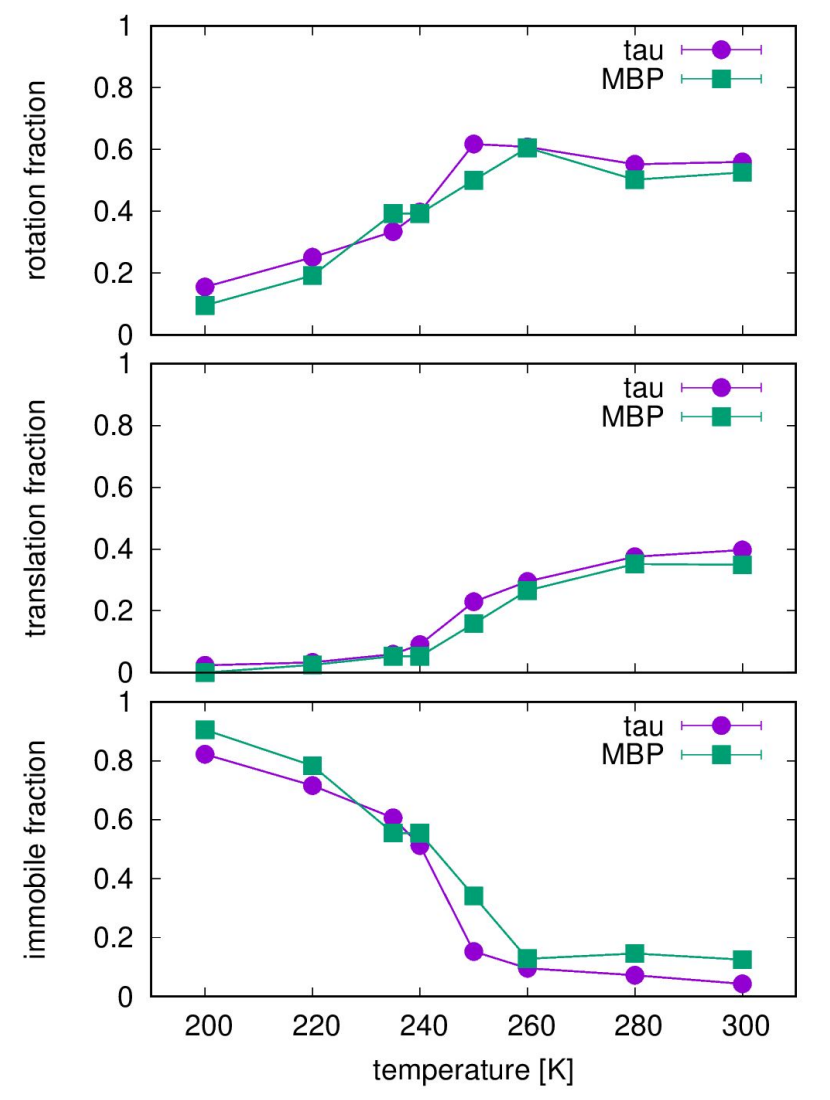

Fig. 5. Fractions of the different components of water dynamics in both tau (purple circles) and MBP (green squares) extracted from the analysis of quasi-elastic spectra as a function of temperature: water molecules not moving in the dynamic window investigated (lower panel), centre-of-mass translation of water molecules (mid panel) and rotation of water molecules around their centre-of-mass (upper panel). An onset of translational motions is evident at $\sim 240 \mathrm{~K}$, while rotational motions are present already at 200 K. Data extracted from [6].

The experimental work reviewed in the present section proved the notion already proposed by molecular dynamics simulations [17,18] that the protein dynamical transition detected by neutron scattering at $\sim 240 \mathrm{~K}[12]$ is connected to the onset of water translational diffusion via 
relaxation of the protein-water hydrogen bond network. Molecular dynamics simulations on the same systems studied by neutron scattering were also used to confirm the experimental results in [6], but this computational part will not be reviewed here. An essential role in the present approach was played by the line width analysis of quasi-elastic data that provided insight into the nature of motions of water molecules in the hydration layer not provided by elastic neutron scattering [13]. The fact that both the globular protein MBP and the intrinsically disordered protein tau display the same mechanism suggested, unexpectedly, that this connection is independent of the degree of order in the protein conformation, and is a general behavior of soluble proteins.

The same approach by protein perdeuteration and neutron scattering was applied to the study of the dynamics of human tau when its amyloid pathological form is induced [7]. The biological function of human tau is the regulation of microtubule stability in neurons. In the case of Alzheimer disease, tau aggregates and forms so-called paired helical filaments (PHF) [19]. Quasi-elastic neutron scattering spectra were measured on both native and aggregated deuterated tau at $280 \mathrm{~K}$. Aggregated tau displays a larger quasi-elastic broadening, in qualitative agreement with the enhanced dynamics of hydration water evidenced in the same sample by elastic neutron scattering. Quasi-elastic data were fitted with the same approach described above [6]. The fraction of water molecules undergoing translational diffusion around the tau PHF was found to be $\sim 25 \%$ higher than around native tau. The translational diffusion coefficient and the rotational rate were $\sim 11 \%$ and $\sim 17 \%$ higher, respectively, for the PHF hydration water. These results provided experimental evidence that hydration water mobility of tau increases upon amyloid aggregation. Such an increase of water dynamics on the surface of tau PHF suggested a scenario in which the hydration water mobility favors the formation of tau amyloid fibers by providing entropic compensation to the disorder-order (from the intrinsically disordered conformation to the PHF alignment) transition induced in the protein during PHF growth.

\subsection{Inelastic neutron scattering probes collective dynamics of lipid membranes}

Understanding the physical properties of lipid membranes is one of the main objectives of biophysical sciences. The use of model systems for lipid bilayers is aimed at obtaining information on the behavior of cell membranes in physiological conditions. In particular, the structural organisation of membranes is supposed to determine their collective response to cellular interactions. The collective dynamics of lipid molecules forming a membrane affect significantly its physical properties. In the cellular context, collective motions are involved in different biological functions. As an example, the transport of small molecules through the membrane needs correlated molecular motions of the lipid acyl chains and the corresponding density fluctuations in the membrane plane [20]. This kind of short range collective motions can be studied by inelastic neutron scattering. One of the first inelastic studies, of which some results are reviewed in this section, focused on the collective dynamics of the lipid acyl chains in the model system 1,2-dimyristoyl-sn-glycero-3-phoshatidylcholine (DMPC)-d54 [8]. Using selective deuteration of the chains to enhance the respective motions over other contributions to the inelastic scattering cross section, the dynamic structure factor $S(Q, \omega)$ was measured in both the gel and the fluid phase of the DMPC bilayer. The temperature dependence of the excitations in the dispersion minimum was also investigated in the vicinity of the main phase transition, occurring at $\mathrm{T}_{\mathrm{c}}=21.5^{\circ} \mathrm{C}$ in the deuterated compound. The experiments were performed at the spectrometers IN12 and IN3 at the Institut Laue-Langevin (ILL) in Grenoble, France. The probe energy was of the same order as the excitations $(\mathrm{meV})$ and the energy resolution $\sim 300$ 
$\mu \mathrm{eV}$. The direction of the scattering vector $\widehat{Q}$ was in the membrane plane in order to use the same experimental set-up to measure both the static structure factor $S\left(Q_{r}\right)$, where $Q_{r}$ is the lateral momentum transfer, and the dynamic structure factor $S\left(Q_{r}, \omega\right)$.

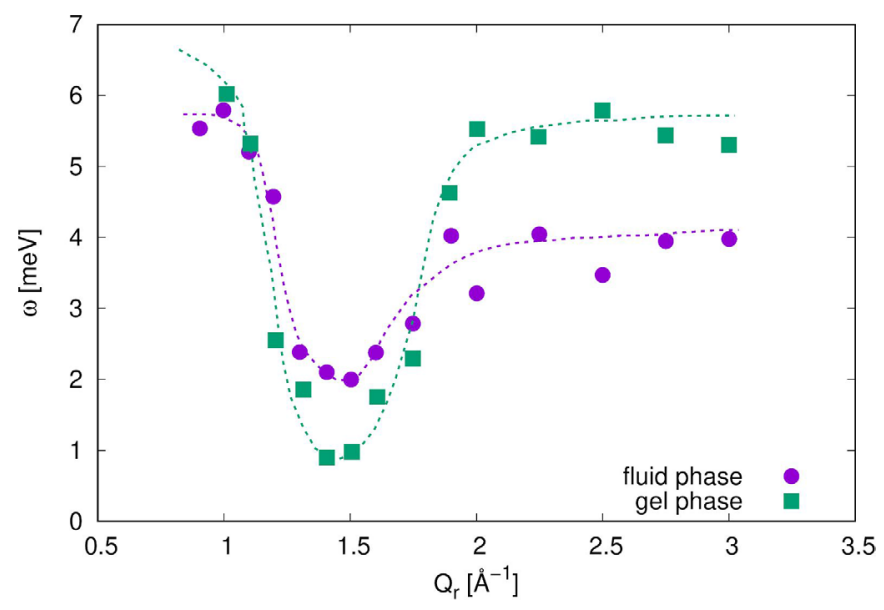

Fig. 6. Dispersion relations in both the fluid $\left(\mathrm{T}=30^{\circ} \mathrm{C}\right.$, purple circles) and the gel phase $\left(\mathrm{T}=20^{\circ} \mathrm{C}\right.$, green squares) of the DMPC bilayer. The energy at the minimum is lower for the gel phase than for the fluid phase. Data extracted from [8].

Using the energy positions of the Brillouin peaks in the inelastic scans at different $Q$ values in the range $0.7<Q<3.0 \AA^{-1}$ the dispersion relation in both the gel and the fluid phase was determined (Fig. 6). The results in Fig. 6 show that the energy at the minimum of the dispersion relation is actually deeper in the gel than in the fluid phase. From the biological point of view, determining the dispersion relation in both phases can help understanding how molecules can be transported across to as well as along the plane of the lipid bilayer, in particular when considering that the collective dynamics at the level of the lipid tails may couple with the self-diffusive dynamics of the lipid molecules. Free area enabling diffusive dynamics can indeed be induced by the collective density waves detected in [8], with the wave frequency providing the vibrational bath for thermally activated conformations of the lipid chains which enable the formation of free volume.

\subsection{High-resolution neutron scattering simultaneously probes internal and diffusive protein dynamics in crowded conditions}

Biological molecules in vivo (i.e. in the cellular environment) are characterized by conditions of crowding and geometrical confinement [21]. Protein and RNA concentration inside the cell is of the order of $\sim 300-400 \mathrm{mg} / \mathrm{ml}$, corresponding to about $30 \%$ of the cytoplasm volume. For example, the concentration of hemoglobin alone is $\sim 350 \mathrm{mg} / \mathrm{ml}$ inside the red blood cells. Several works (see e.g [22] and references therein) showed that chemical equilibria and chemical reactivity inside the cell are perturbed by non-specific solute-solute interactions and excluded volume effects. However, studies on the dynamical properties of proteins in crowded conditions are still not very abundant. In the last years quasi-elastic neutron backscattering at 
the spectrometers IN16 and IN10 (ILL, Grenoble) has been used to obtain extensive experimental data on protein self-diffusion on the nanosecond time and nanometer length scales in crowded aqueous solutions of bovine serum albumin (BSA) [9], $\beta$-lactoglobulin (BLG) [10] and other proteins at high concentration [23].

In the first work on BSA [9], quasi-elastic spectra were modeled by a convolution of two lorentzian terms, one accounting for fast internal and interdomain motions within the protein and the other one for the convolution of the translational and rotational diffusion of the entire protein. From the $Q$-dependence of the linewidth of the second term the total diffusion coefficient $D$ was determined in the low concentration extrapolation and compared with the same parameter obtained by dynamic light scattering to validate the approach. An analytical method was then developed to extract the translational diffusion coefficient $D_{t}$ from $D$ also for the high concentration regime. The study of $D_{t}$ as a function of the protein volume fraction showed that crowding has a strong effect on the protein self-diffusion already on the nanoseconds time scale, the diffusion coefficient at high protein concentration being strongly decreased compared to the dilute limit. Such a slowing-down effect on the protein diffusion was found to be mainly caused by hydrodynamic interactions since it could be described with very good accuracy in terms of colloidal short-time self-diffusion.
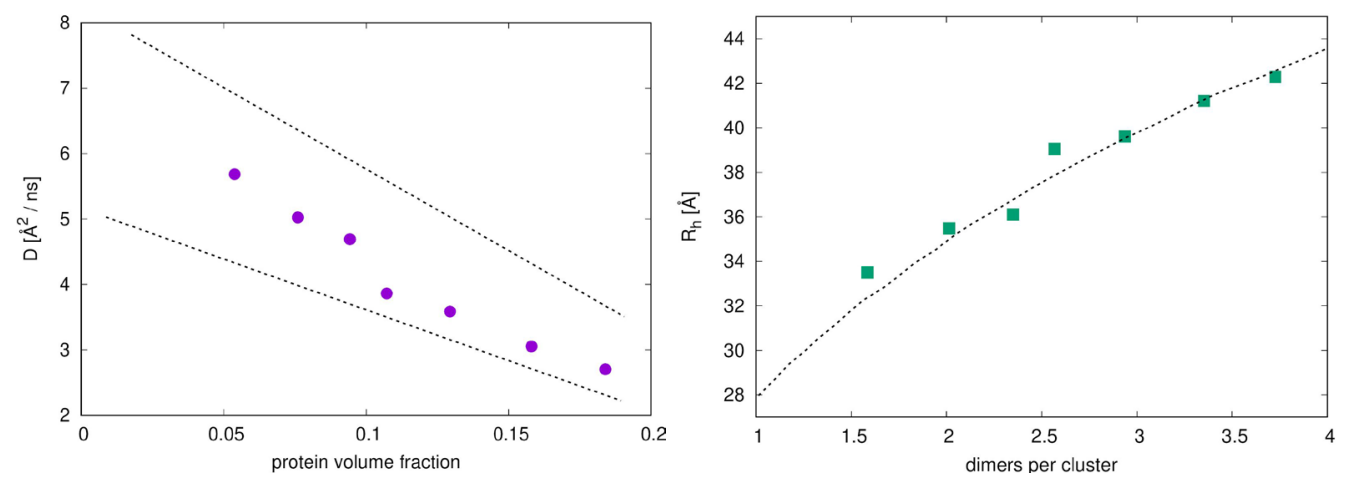

Fig. 7. Left panel: apparent diffusion coefficients $D$ (purple circles) of BLG obtained by the analysis of neutron backscattering data as a function of the protein volume fraction. The black dotted lines are calculated considering the diffusion coefficient of a BLG dimer (upper line) and a cluster of four BLG dimers (lower line). Right panel: effective hydrodynamic radii $R_{h}$ (green squares) of the BLG clusters as a function of the number of dimers per cluster calculated from small angle X-ray scattering data. The black dotted line is a fitting curve with a function $R_{h} \propto N^{a}$ yielding $a=0.32 \pm 0.02$. Data extracted from [10].

The second work reviewed in this section [10] used an analogous approach to focus on the clustering of BLG dimers in crowded conditions. The phenomenon of clustering in protein aqueous solutions is interesting from both a fundamental and a practical point of view. The mechanisms inducing cluster formation have been investigated in model systems, yet their understanding may help to control processes like protein self-assembly, drug delivery, protein aggregation and crystallization. In BLG solutions clustering can be induced just by increasing the protein volume fraction, without the necessity for changing other chemical parameters like 
salt concentration or $\mathrm{pH}$. In physiological conditions and at low volume fraction BLG is found predominantly as a dimer.

A detailed description of cluster formation was obtained from the self-diffusion coefficients measured by neutron backscattering as a function of protein volume fraction in the range 0.05 0.2 (Fig. 7 left panel). In the high concentration regime, the apparent diffusion coefficients were not in agreement with those calculated assuming the presence of BLG dimers while a better agreement was obtained with BLG clusters formed by four dimers (dotted lines in Fig. 7 left panel). More precise information on the average hydrodynamic size of the clusters was derived from a fitting of the apparent diffusion coefficients $D$ at each different protein concentration using the effective hydrodynamic radius $R_{h}$ as only free parameter (Fig. 7 right panel). A scaling law $R_{h} \propto N^{a}$ was used to fit the data (Fig. 7 right panel), with the fitting exponent found to be $a=0.32 \pm 0.02$. An exponent $\sim 1 / 3$ is consistent with the presence of quite compact clusters of BLG dimers whose size increases with increasing protein concentration. The combination of backscattering results with complementary data from small angle X-ray scattering and neutron spin echo (not described here) enabled the authors to obtain a robust description of the presence of clusters in BLG aqueous solutions that are static on the nanosecond time scale accessed by neutron spectroscopy. The analysis proposed in [10] can be used as a general approach to provide a quantitative description of the structure and the diffusive dynamics of macromolecular assemblies at high concentration.

\section{References}

1. S. W. Lovesey, Theory of Neutron Scattering from Condensed Matter (Clarendon Press, Oxford, 1984)

2. G. L. Squires, Introduction to the Theory of Thermal Neutron Scattering (Courier Corporation, 1996)

3. M. Bée, Quasielastic Neutron Scattering (Adam Hilger, 1988)

4. E. Johnson and L. Robinson, Rev. Sci. Instrum. 60, 3447 (1989)

5. L. Van Hove, Phys. Rev. 95, 249 (1954)

6. G. Schirò, Y. Fichou, F.-X. Gallat, K. Wood, F. Gabel, M. Moulin, M. Härtlein, M. Heyden, J.-P. Colletier, A. Orecchini, A. Paciaroni, J. Wuttke, D. J. Tobias, and M. Weik, Nat. Commun. 6, 6490 (2015)

7. Y. Fichou, G. Schirò, F.-X. Gallat, C. Laguri, M. Moulin, J. Combet, M. Zamponi, M. Härtlein, C. Picart, E. Mossou, H. Lortat-Jacob, J.-P. Colletier, D. J. Tobias, and M. Weik, Proc. Natl. Acad. Sci. U. S. A. 112, 6365 (2015)

8. M. C. Rheinstädter, C. Ollinger, G. Fragneto, F. Demmel, and T. Salditt, Phys. Rev. Lett. 93, 108107 (2004)

9. F. Roosen-Runge, M. Hennig, F. Zhang, R. M. J. Jacobs, M. Sztucki, H. Schober, T. Seydel, and F. Schreiber, Proc. Natl. Acad. Sci. U. S. A. 108, 11815 (2011)

10. M. K. Braun, M. Grimaldo, F. Roosen-Runge, I. Hoffmann, O. Czakkel, M. Sztucki, F. Zhang, F. Schreiber, and T. Seydel, The Journal of Physical Chemistry Letters 8, 2590 (2017)

11. G. Schirò and M. Weik, Journal of Physics: Condensed Matter 31, 463002 (2019)

12. W. Doster, S. Cusack, and W. Petry, Nature 337, 754 (1989)

13. K. Wood, A. Frölich, A. Paciaroni, M. Moulin, M. Härtlein, G. Zaccai, D. J. Tobias, and M. Weik, J. Am. Chem. Soc. 130, 4586 (2008)

14. T. Kleinert, W. Doster, F. Post, and M. Settles, in Conference Proceedings-Italian Physical Society (Editrice Compositori, 1992), pp. 127-127 
15. S. K. Pal, J. Peon, and A. H. Zewail, Proc. Natl. Acad. Sci. U. S. A. 99, 1763 (2002)

16. G. Schirò, M. Fomina, and A. Cupane, J. Chem. Phys. 139, 121102 (2013)

17. M. Tarek and D. J. Tobias, Phys. Rev. Lett. 88, 138101 (2002)

18. A. L. Tournier, J. Xu, and J. C. Smith, Biophys. J. 85, 1871 (2003)

19. K. S. Kosik, C. L. Joachim, and D. J. Selkoe, Proc. Natl. Acad. Sci. U. S. A. 83, 4044 (1986)

20. S. Paula, A. G. Volkov, A. N. Van Hoek, T. H. Haines, and D. W. Deamer, Biophys. J. 70, 339 (1996)

21. R. J. Ellis, R. John Ellis, and A. P. Minton, Nature 425, 27 (2003)

22. A. P. Minton, Journal of Biological Chemistry 276, 10577 (2001)

23. M. Grimaldo, F. Roosen-Runge, F. Zhang, F. Schreiber, and T. Seydel, Quarterly Reviews of Biophysics 52, (2019) 